\title{
Columellar Aesthetics in Open Rhinoplasty
}

\author{
Russell W.H. Kridel, MD ${ }^{1}$ Edward S. Kwak, MD ${ }^{2}$ Jeffrey B. Watson, MD ${ }^{1}$ \\ ${ }^{1}$ Division of Facial Plastic Surgery, Department of Otolaryngology - \\ Head and Neck Surgery, University of Texas Health Sciences Center, \\ Houston, Texas \\ Address for correspondence Russell W.H. Kridel, MD, Facial Plastic \\ Surgery Associates, 6655 Travis St., Suite 900, Houston, TX 77030 \\ (e-mail: Russell.W.Kridel@uth.tmc.edu).
}

2 Otolaryngology - Head and Neck Surgery at Icahn School of Medicine at Mount Sinai, New York, New York

Facial Plast Surg 2016;32:333-338.

Abstract
Keywords
- rhinoplasty
- open rhinoplasty
- columella
- columellar aesthetics
- columellar
deformities
- columellar
disproportion
- nostril shape

Detailed descriptions of the ideals of columellar aesthetics and nostril shape are conspicuously lacking from the medical literature. Achieving an aesthetic nasal base is critical to an optimal rhinoplasty result. Deviations in the columella and variations in its width and height lead to distortion of nostril shape and frequently compromise function. Six types of columellar disproportion are presented with detailed explanations of how to treat each. By properly recognizing and addressing columellar deformities, the surgeon can optimize both the aesthetic and functional results that are achieved.
The aesthetically appealing nose unites individual subunits to create a harmonious and functional figure. Transitions between nasal subunits are seamless, with each subunit complementing those adjacent to it. One region of the nose where these principles hold particularly true is the nasal base. The nasal base is composed of the nostrils, the alae, the lobule, the nasal sills, and the columella. Each of these components contributes to the overall shape of the nasal base and appropriate proportions are paramount. The nasal columella, in particular, has a pivotal role in determining the shape of the nasal base. Deviations in the columella and variations in its width and height lead to distortion of nostril shape and frequently compromise function.

\section{Anatomy and Aesthetic Proportions of the Columella}

The columella begins at the apex of the nostril, inferior to the lobule, and extends inferiorly until it transitions laterally into the nasal sill. The cartilaginous and, to a lesser degree, bony framework of the columella is composed of paired medial crura, caudal septal cartilage, and the nasal spine. Soft-tissue components contribute to the shape of the columella and include the septal membranes, depressor nasi septi muscle, and the intervening soft tissue between the medial crura.

To date, descriptions of the aesthetic columellar ideals are limited and there is a paucity of literature examining the various configurations of columellar shapes. ${ }^{1}$ First and foremost, one must recognize that the aesthetic columellar shape should not itself be the focal point of the nasal base; rather, attention should be drawn to achieving symmetry between the nostrils and understanding how the shape of the columella influences this. The ideal columella starts at the apex of the nostrils with a width approximately equal to that of the tip defining points. The columella then tapers symmetrically reaching its narrowest width approximately at the junction between the middle one-third and lower one-third of the height of the columella. At this point, the columella starts to gradually broaden. At the base of the columella, the width is slightly greater than at its apex. The transition to the nasal sill is gentle but distinguishable (-Fig. 1).

The overall ideal aesthetic nasal base has been described as having the shape of an equilateral triangle. ${ }^{2}$ The height of the columella should be two-thirds the height of the base and
Issue Theme Challenging Problems in Rhinoplasty; Guest Editor, Hossam M.T. Foda, MD
Copyright (c) 2016 by Thieme Medical Publishers, Inc., 333 Seventh Avenue, New York, NY 10001, USA. Tel: +1(212) 584-4662.
DOI http://dx.doi.org/ 10.1055/s-0036-1585575. ISSN 0736-6825. 


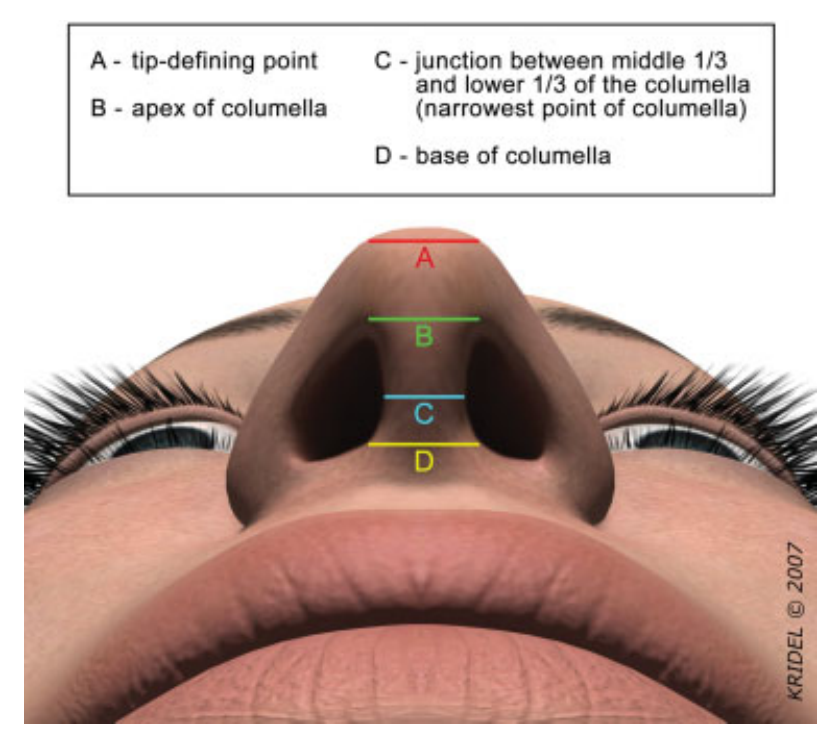

Fig. 1 The columella begins at the apex of the nostrils $(B)$, then tapers to its narrowest point at the junction of the middle and lower onethirds $(C)$ before gradually broadening as it transitions to the nasal sill (D). (Copyright and reprinted with permission from Russell W.H. Kridel.)

therefore twice the height of the lobule. ${ }^{1,3,4}$ Clinically, the shape of the columella varies considerably. A short columella is seen in noses with inadequate tip projection and weak medial crura. The columella to lobule ratio may approach 1:1. The medial crura may splay at the base of the columella adding excess width, causing the nostrils to have a rounder appearance rather than the more favorable oblique teardrop configuration (-Fig. 2A, B).

A triangular shaped columella is seen when an extremely wide columella extends to the alar-facial groove, resulting in slit-like nostril shapes. Frequently, this is caused by medial crura that diverge increasingly as they extend inferiorly and approach the corner of the nasal sill ( - Fig. 3A, B).

\section{Functional Implications of Columellar Shape}

\section{External Nasal Valve}

The external nasal valve is defined as the nasal aperture caudal to the internal nasal valve. ${ }^{2}$ Commonly, the external

\section{A}

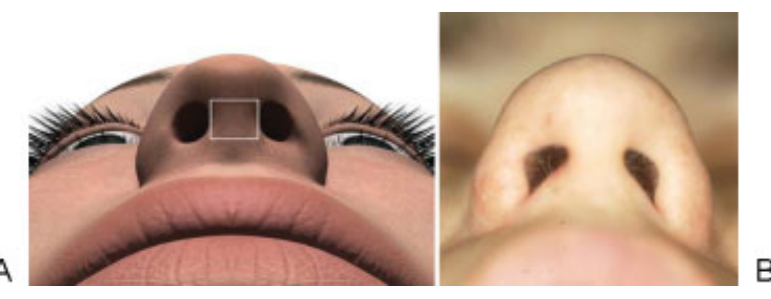

Fig. 2 (A, B) A square-shaped columella is seen in noses with inadequate tip projection and weak medial crura. Besides lacking adequate height, there is excess width of the columella, almost equal to the height. At the base of the columella, the medial crura are frequently splayed. (Copyright and reprinted with permission from Russell W.H. Kridel.)

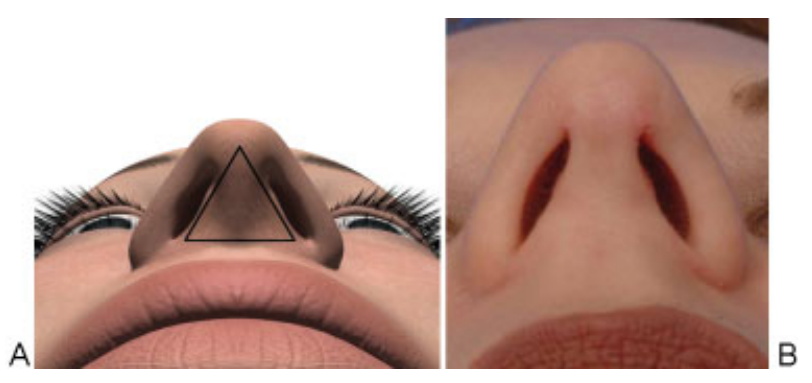

Fig. 3 (A, B) A triangle-shaped columella is seen when a wide columella extends to the alar-facial groove resulting in slit-like nostril shapes. (Copyright and reprinted with permission from Russell W.H. Kridel.)

nasal valve is thought of as the mobile alar sidewall in isolation; however, it is important to recognize that the external valve includes the cutaneous, soft tissue, and cartilaginous framework of not only the ala but also the columella and caudal septum. Addressing external valve collapse therefore includes supporting not only the ala through the use of alar rim and batten grafts but also addressing columellar and caudal septal deformities. Prior studies have highlighted the implications of a wide columella as it pertains to the function of the external valve. ${ }^{5,6}$ A significantly wide columella with splayed medial crura may extend across the nasal sill as far as the alar-facial insertion, creating a narrow slit-like shape to the nostril and significantly decreasing airflow to the nasal cavity ( - Fig. 4). Narrowing the columella in these situations will increase the nasal aperture and dramatically improve nasal airflow for the patient. Failure to recognize the shape of the columella as a component of the external valve can potentially result in nasal obstruction that persists following functional nasal surgery.

\section{Examination of the Columella}

A complete nasal evaluation is performed and photo-documented. Included in the examination is evaluation of the
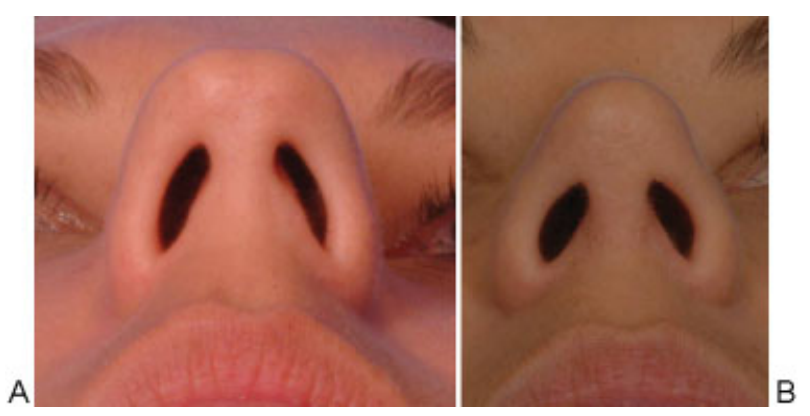

Fig. 4 (A) Preoperative view demonstrating a wide columella extending to the alar-facial insertion, narrowing the external nasal valve and decreasing airflow to the nasal cavity. (B) Nine months postoperative. After narrowing the columella, the patient noted improved nasal airflow. (Copyright and reprinted with permission from Russell W.H. Kridel.) 
external nasal valve from the base view, recognizing the potential contribution of a weak alar rim and wide columella to a narrowed valve. The entire structure of the external nasal valve including the shape of the columella should be assessed both passively and actively with deep nasal inspiration. It is critical that before performing anterior rhinoscopy with the speculum, one must examine the columella and alae to assess whether the external valve may be restricted. The columella is palpated to assess the position and shape of the medial crura, soft-tissue fullness between the medial crura, and the relative length and position of the caudal septum. Columellar skin thickness is also appreciated as thin skin may contribute to the visible presence of bifidity.

On profile view, the alar-columellar relationship is assessed for columellar show or alar retraction. The patient is asked to smile to determine whether there is depressor nasi septi function that causes undesirable counter-rotation of the nasal tip when smiling. The tip is also palpated to determine the degree of support.

In revision cases, a wide columella may be due to numerous causes and it is helpful to try to assess the reason preoperatively. Previously placed columellar struts, tip grafts, plumping grafts, and caudal septal extension grafts may all contribute to the shape of the columella. All medical records and operative reports from previous nasal surgeries are evaluated when available.

\section{Recognizing and Addressing the Six Types of Columellar Disproportion}

\section{Caudal Septal Deviation}

Owing to its rigidity, size, and position, the caudal septal cartilage functions as the foundational framework of the nose, with its shape significantly impacting adjacent structures. The normal caudal septum stops just posterior to the columella, and the membranous septum spans the short distance anteriorly to the medial crura and columella. When the caudal septum is overdeveloped, it may extend into the columella where it can interpose between the medial crura or push one of the medial crural footplates to one side. This results in asymmetric or unilateral columellar width and secondary asymmetry of the nostrils (-Fig. 5). When caudal septal deviation is recognized as a component of a wide columella, it should be addressed first. Once the septum is

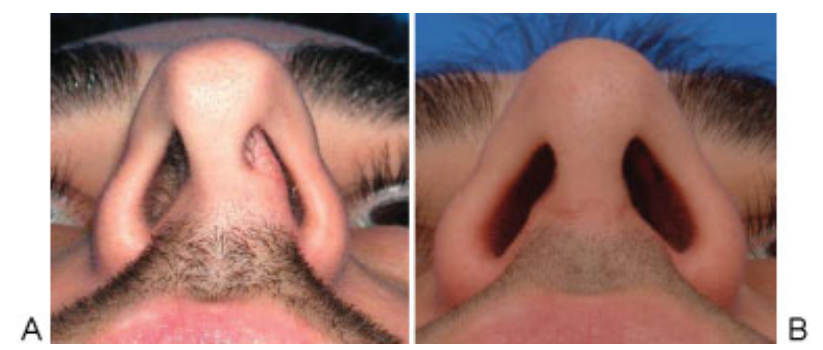

Fig. 5 (A, B) A severely deviated septum can cause asymmetric splay of the medial crural footplates to one side and increased columellar width. (A) Preoperative view. (B) Three months postoperative view. (Copyright and reprinted with permission from Russell W.H. Kridel.) set to midline, the relative contribution of the surrounding structures can be more accurately addressed.

To address a caudal septal deviation, it is recommended that a hemi- or full-transfixion incision be utilized to access the septum. The incision should be placed on the side of the deviation to allow excision of what will become redundant skin and mucosa after the deviation is corrected. A fulltransfixion incision is not always required to correct caudal septal deviations except in cases of deviation where both sides of the membrane must be elevated to allow the crooked caudal septum to be straightened. Once the mucoperichondrial flaps are elevated and the septal cartilage is isolated, any posterior portion of the septum that is deviated is removed, preserving an adequate caudal and dorsal strut. The first step is accomplished by disarticulating the cartilaginous septum at the junction of the quadrangular septal cartilage and bony vomer. Then, inferiorly, the deviated septal cartilage should be separated off the maxillary crest working posterior to anterior toward the nasal spine. To achieve full mobility of the septum, often times, a small amount of septal cartilage is trimmed inferiorly over the crest, slightly shortening the height of the inferior septum to allow it to swing back to midline. For caudal deviations, the caudal septum must be repositioned to the midline. The caudal septum is secured in the midline with 5-0 PDS (Polydioxanone; Ethicon Inc., Somerville, NJ) or vicryl (Polydioxanone; Ethicon Inc.) suture passed through the periosteum overlying the nasal spine.

If the septum continues to lean to the side despite the preceding procedure, one may use the tongue-in-groove technique to allow the septum to sandwich between the medial crura in the midline. ${ }^{7}$ A retrograde dissection is performed between the medial crura using tenotomy scissors, creating a pocket into which the caudal septum may extend. Because of the potential space created between the medial crura, trimming septal caudal cartilage is not usually necessary. Any other contributing factors to excess columellar width are then addressed. Frequently redundant septal membrane is removed bilaterally posterior to the transfixion incision, which acts as a hammock to hold the columellar correction in place. Lastly, 4-0 chromic gut septocolumellar sutures are placed through the septum, medial crura, and septal membranes to secure the septum between the medial crura during the healing process.

\section{Medial Crural Footplate Splay}

In a patient with a wide columella who has a midline caudal septum or in whom the septum has been reset to midline per the preceding procedure, the columellar shape can then be inspected for medial crural footplate splay. Excessive splay of the footplates is palpable and springlike on examination. It may be due to excess convexity of the medial crural footplates or due to short medial crura in which the footplates diverge at the midcolumella instead of the base, thus causing excess width where not desirable (-Fig. 6A-C). To address this problem, the medial crural footplates can be approached via a hemi- or full-transfixion incision. The medial surface of each medial crural footplate can be scored using a no. 15 
A
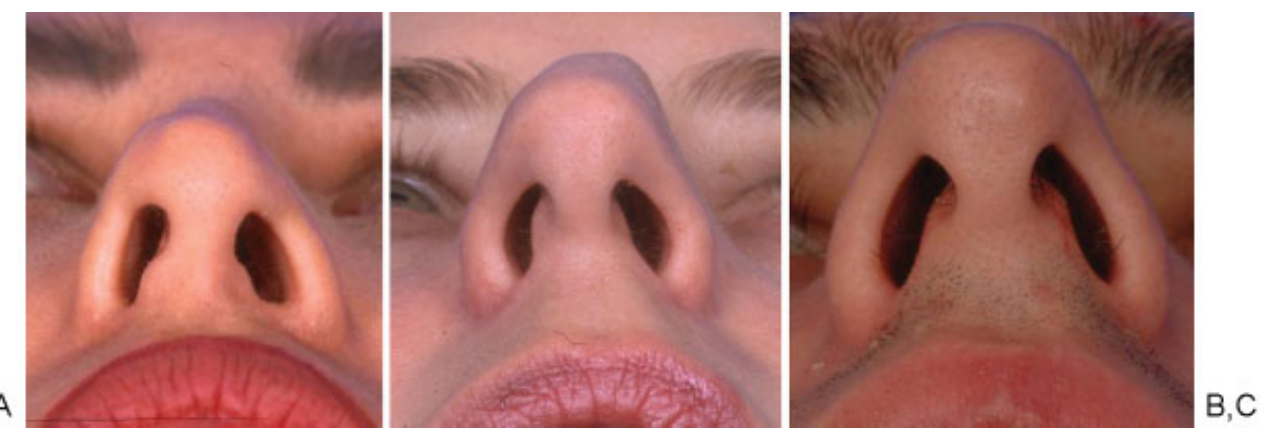

Fig. 6 (A-C) The length of the medial crura and the amount of splay between the two opposing sides is clinically variable and affects the width and shape of the columella. (Copyright and reprinted with permission from Russell W.H. Kridel.)

blade or fine scissors to diminish the cartilage memory. The footplates may then be stabilized in a more narrow and straight configuration by suturing them together or to a columellar strut. The sutures should be placed within the inferior half of the medial crura so as to not overnarrow the superior portion of the columella where it transitions to the broader lobule. Suture fixation also helps to straighten and strengthen the medial crura in cases where they may be weak or misshapen. If an endonasal approach is utilized, the footplates may be secured with 6-0 polypropylene sutures via the transfixion incision.

\section{Excessive Soft Tissue and the Depressor Nasi Septi}

Variable amounts of soft tissue are found between the medial crura and affect the width of the columella accordingly. The two primary soft-tissue structures found within the columella are the depressor nasi septi and the levator nasi septi. ${ }^{8}$ The depressor nasi septi is a paired muscle originating in the incisive fossa of the maxillary canine tooth and fanning out to insert on the inferior portion of the medial crura, floor of the nostril, septal membrane, and inner surface of the ala of the nose ( - Fig. 7). In patients who have clinically evident activity of the muscle, smiling results in descent of the nasal tip,

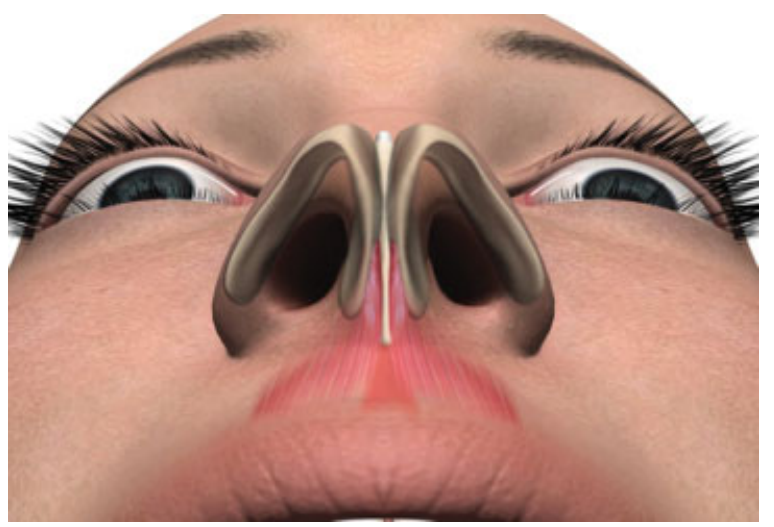

Fig. 7 The depressor nasi septi is a paired muscle originating in the incisive fossa of the maxillary canine tooth and fanning out to insert on medial crura, floor of the nostril, septal membrane, and inner surface of the ala of the nose. (Copyright and reprinted with permission from Russell W.H. Kridel.) shortening of the upper lip, and widening of the columella. ${ }^{9}$ The muscle may be resected to prevent this action or in cases where muscle bulk is contributing to a wide columella.

Prior investigation has demonstrated that the levator nasi septi muscle contributes, in part, to the columellar soft tissue and is present in varying degrees among patients. ${ }^{8}$ Though not anatomically distinct from the depressor muscle during rhinoplasty, a portion of this muscle may be resected during soft-tissue removal between the medial crura.

The soft tissue and muscle complex between the medial crura is approached via a full-transfixion incision that allows access to bilateral muscle insertions. Scissors are used to retrodissect between the medial crura. A single hook is then inserted under the vestibular skin along the lateral aspect of the medial crus allowing the space between the medial crura to open and the soft tissue and muscle to be visualized (-Fig. 8). The tissue is resected with needle point electrocautery. To hold the medial crura together and prevent widening during healing, 4-0 chromic gut mattressed septocolumellar sutures are placed. Although a few patients may have temporary diminished elevation of the central lip after depressor nasi septi resection, we have not experienced any patient with permanent changes to the smile after medial crural softtissue resection. Resection of the depressor nasi septi muscle leads to consistent termination of tip ptosis with smiling and typically is met with very high patient satisfaction.

\section{Deviated Nasal Spine}

A less common contributor to excess columellar width is the nasal spine, which in some patients may be off-center or wide. The nasal spine is typically a small triangular extension of bone in the midline position that develops from fusion of the maxillary alveolar processes. When properly aligned, the anterior-inferior caudal septum rests upon the nasal spine with both structures in the midline and neither adding excess width to the columella. Rarely, however, the nasal spine may be found to be significantly prominent, wide, and/or deviated and therefore causing excess columellar width. In these cases, a lateral nasal spine reduction is performed. Using a 3-mm osteotome, the spine is reduced by making a tangential ostectomy in an anterior-posterior plane along the prominent sidewall of the spine, thereby reducing the width while preserving the majority of the nasal spine in the midline. 


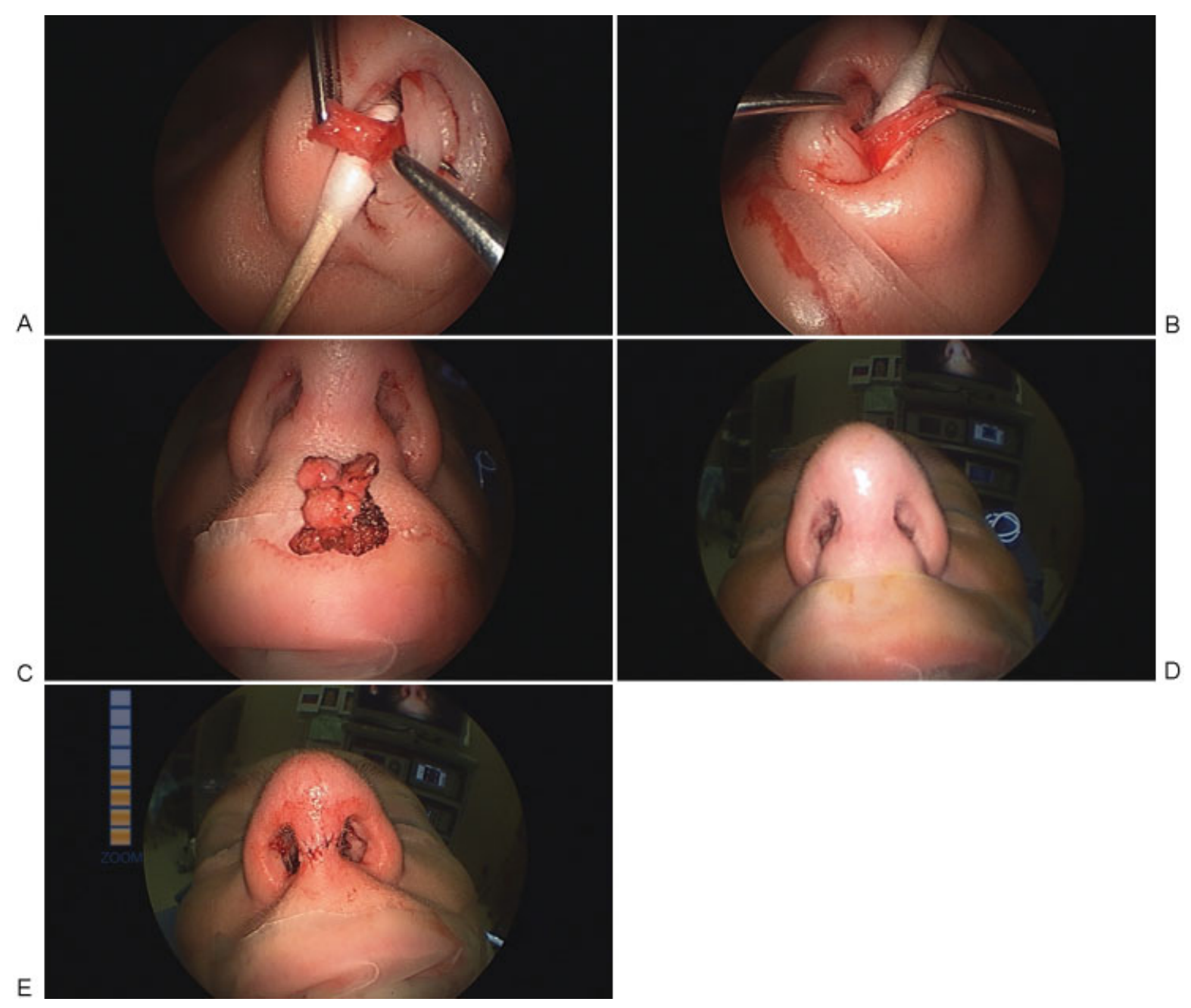

Fig. 8 (A-C) The depressor nasi septi and soft tissue between the medial crura are approached and resected bilaterally via a full-transfixion incision with the aid of a single hook for exposure. (D, E) Pre- and postoperative views. Significant narrowing of the columella can be appreciated at the conclusion of surgery. (Copyright and reprinted with permission from Russell W.H. Kridel.)

\section{latrogenic}

Iatrogenic causes of a widened columella most frequently occur as a result of prominent grafts or scarring due to prior rhinoplasty. As the skin and soft-tissue envelope is elevated, excess scar is removed to expose the underlying structure of the lower lateral cartilages. Grafts that are encountered may be removed or trimmed. Addressing the cartilaginous contributions to the wide columella will allow the skin envelope to redrape with a favorable resulting shape.

\section{Membranous Septum Excision}

Patients with a wide columella often have redundant septal membrane mucosa and excess columellar skin. ${ }^{7}$ This becomes apparent after any combination of the aforementioned techniques has been performed to narrow the internal structure of the columella, thus causing a relative excess of skin and membrane when redraped over the narrower construct. Each side of the nose should be evaluated separately to determine the amount of septal membrane excision needed. A greater amount of septal membrane excision is necessary on the side of a caudal septal deviation or splayed medial crus to keep the membrane taught. The effect of membrane excision is threefold, serving to remove redundant membrane contributing to excess width, preventing flaring of the columellar sidewalls by functioning as a sling, and furthermore correcting and/or preventing a hanging columella resulting from septal membrane excess. To perform this maneuver, the membrane is excised along the transfixion incision in a vertical, fusiform manner (-Fig. 9). It is important to remove tissue from the posterior side of the transfixion incision. This results in vestibular skin advancing into the nose as opposed to bringing moist respiratory mucosa onto the columella and where it may become irritated and wet. If a hemi-transfixion incision rather than full-transfixion incision has been performed, the contralateral side can be addressed with a fusiform excision of redundant septal membrane mucosa in the approximate position of a transfixion incision. Both incisions are closed with simple interrupted 5-0 plain gut sutures. 


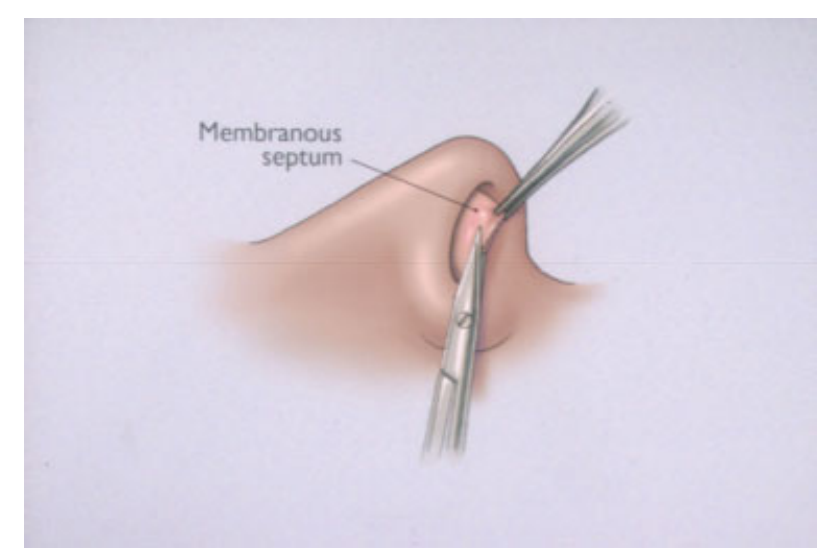

Fig. 9 Membranous septum excision is performed in fusiform fashion along the transfixion incision, taking care to remove tissue from the posterior not anterior side of the incision. (Copyright and reprinted with permission from Kridel et al. ${ }^{7}$ )

\section{Summary of Techniques}

Each of the aforementioned techniques may be applied in isolation or in combination, as required, to address the underlying problems. For the reasons cited previously, we frequently perform membranous septum excision in cases where excess columellar width has been addressed. When seeking to achieve a more aesthetic and narrow columella, most of the changes required are to the middle and base of the columella, as described previously. Changes associated with the apex of the columella are a result of tip refining techniques. Appropriately establishing tip width and definition will secondarily result in appropriate width of the apex of the columella. Changes to the middle and base of the columella can be affected through the direct methods described previously. Finally, the columellar base should transition smoothly to the nasal sill and should therefore be narrowed conservatively. By following the aforementioned techniques in correctly identifying and treating columellar deformities, the surgeon will optimize both the aesthetic and functional results achieved.

\section{References}

1 Tardy ME. Rhinoplasty: The Art and the Science. Philadelphia, PA: W.B. Saunders; 1997

2 Papel I, ed. Facial Plastic and Reconstructive Surgery. 2nd ed. New York, NY: Thieme; 2002

3 Sheen JA, Sheen AP. Aesthetic Rhinoplasty. Saint Louis, MO: Quality Medical Publishing; 1998

4 Powell N, Humphreys B. Proportions of the Aesthetic Face. New York, NY: Thieme-Stratton; 1984

5 Fanous N. Columelloplasty for the wide obstructing columella. Laryngoscope 1990;100(2, Pt 1):203-205

6 Ghidini A, Dallari S, Marchioni D. Surgery of the nasal columella in external valve collapse. Ann Otol Rhinol Laryngol 2002;111(8): 701-703

7 Kridel RW, Scott BA, Foda HM. The tongue-in-groove technique in septorhinoplasty. A 10-year experience. Arch Facial Plast Surg 1999;1(4):246-256, discussion 257-258

8 Song R, Ma H, Pan F. The "levator septi nasi muscle” and its clinical significance. Plast Reconstr Surg 2002;109(5):1707-1712, discussion 1713

9 Rees TD, ed. Rhinoplasty anatomy. In: Aesthetic Plastic Surgery. Philadelphia, PA: W.B. Saunders; 1980:53-65 